\title{
The Molecular Chaperone Apolipoprotein J/Clusterin as a Sensor of Oxidative Stress: Implications in Therapeutic Approaches - A Mini-Review
}

\author{
Ioannis P. Trougakos
}

Department of Cell Biology and Biophysics, Faculty of Biology, University of Athens, Athens, Greece

\section{Key Words}

Ageing · Apolipoprotein J/Clusterin - Cancer - Chaperone ·

Free radicals . Inflammation - Neurodegeneration .

Oxidative stress

\begin{abstract}
Background: Organisms are constantly exposed to physiological and environmental stresses and therefore require an efficient surveillance of genome and proteome quality in order to prevent disruption of homeostasis. Central to the intra- and extracellular proteome surveillance system are the molecular chaperones that contribute to both proteome maintenance and clearance. The conventional protein product of the apolipoprotein J/clusterin (CLU) gene is a heterodimeric secreted glycoprotein (also termed as sCLU) with a ubiquitous expression in human tissues. CLU exerts a small heat shock protein-like stress-induced chaperone activity and has been functionally implicated in numerous physiological processes as well as in ageing and most age-related diseases including tumorigenesis, neurodegeneration, and cardiovascular and metabolic syndromes. Objective: The CLU gene is differentially regulated by a wide variety of stimuli due to the combined presence of many distinct regula-
\end{abstract}

tory elements in its promoter that make it an extremely sensitive cellular biosensor of environmental and/or oxidative stress. Downstream to CLU gene induction, the CLU protein seems to actively intervene in pathological states of increased oxidative injury due to its chaperone-related property to inhibit protein aggregation and precipitation (a main feature of oxidant injury), as well as due to its reported distribution in both extra- and, most likely, intracellular compartments. Conclusion: On the basis of these findings, CLU has emerged as a unique regulator of cellular proteostasis. Nevertheless, it seemingly exerts a dual function in pathology. For instance, in normal cells and during early phases of carcinogenesis, CLU may inhibit tumor progression as it contributes to suppression of proteotoxic stress. In advanced neoplasia, however, it may offer a significant survival advantage in the tumor by suppressing many therapeutic stressors and enhancing metastasis. This review will critically present a synopsis of recent novel findings that relate to the function of this amazing molecule and support the notion that CLU is a biosensor of oxidative injury; a common link between ageing and all pathologies where CLU has been implicated. Potential future perspectives, implications and opportunities for translational research and the development of new therapies will be discussed.

Copyright $\odot 2013$ S. Karger AG, Basel

\section{KARGER}

E-Mail karger@karger.com

www.karger.com/ger
(C) 2013 S. Karger AG, Basel

0304-324X/13/0596-0514\$38.00/0
Ioannis $\mathrm{P}$. Trougakos, $\mathrm{PhD}$

Department of Cell Biology and Biophysics

Faculty of Biology, University of Athens, Panepistimiopolis

GR-15784 Athens (Greece)

E-Mail itrougakos@biol.uoa.gr 


\section{Introduction}

\section{Molecular Effects of Oxidants in Higher Metazoan}

Cells

Oxygen is essential to support aerobic metabolism and life. Nevertheless, in the reductive normal cellular milieu, oxygen molecules undergo univalent reductions forming (among others) 'reactive nitrogen species' and 'reactive oxygen species' (ROS), as well as 'reactive lipid peroxidation products' [1]. Free radicals may arise from both exogenous and endogenous sources. Exogenous environmental sources include gamma rays, X-rays, UV light, atmospheric pollutants and metal catalyzed reactions, whereas endogenous organism sources of free radicals production include various inflammatory processes, as well as the excessive stimulation of $\mathrm{NAD}(\mathrm{P}) \mathrm{H}$ oxidases, mitochondria malfunction, $\mathrm{P} 450$ metabolism and/or the activity of peroxisomes [2].

Oxidative stress occurs when the production of free radicals exceeds a certain threshold needed as a secondary messenger in intracellular signaling cascades, and the cell's antioxidant capacity $[3,4]$. Sustained abnormally high levels of ROS may result in deregulation of redoxsensitive signaling pathways and extensive damage of all types of cellular biomolecules including DNA and proteins [4]. Thus, the function of the antioxidant molecules and of the cellular mechanisms that regulate the ability of the cells and tissues to adapt or to resist to oxidative stress is critical for survival and homeostasis.

A first line of defense against oxidative stress is achieved by the action of the antioxidants (e.g. vitamins $\mathrm{C}$ and $\mathrm{E}$, glutathione, and thioredoxin) and the antioxidant enzymes (e.g. superoxide dismutase, catalase, glutathione peroxidase, glutathione reductase) [4], whereas a second line of defense refers to a network of transcription factors that are activated as a response to increased oxidative stress or cellular oxidative injury. Among these, the evolutionary conserved NF-E2-related factor-2 (Nrf2)/ Kelch-like ECH-associated protein 1 (Keap1) signaling pathway is central in the protection of cells against oxidative and xenobiotic damage [5]. Under normal conditions the actin-associated protein Keap1 binds to and tethers Nrf2 in the cytoplasm, whereas upon oxidative stress, the Keap1/Nrf2 association is disrupted, resulting in the release of $\mathrm{Nrf2}$, which then accumulates in the nucleus where it heterodimerizes with a small Maf (musculoaponeurotic fibrosarcoma) protein, and by binding to antioxidant response elements stimulates the expression of phase II and antioxidant enzymes [5]. Besides Nrf2, oxidative stress can also activate a number of additional nuclear transcription factors including heat shock factor-1 (HSF1), activator protein-1 (AP-1), p53, NF- $\mathrm{BB}$, and signal transducer and activator of transcription (STAT) [2]. Similarly to Nrf2, in the absence of stress, HSF1 is kept inactive in the cytosol mainly by binding to HSP90. Upon oxidative stress, the inhibiting chaperones bind misfolded or damaged proteins; the HSF1 is liberated and by translocating to the nucleus it activates several target genes [6]. AP-1 or p53 activation by oxidative or other physical and chemical stressors trigger the activation of numerous processes involved in many different stress responses [7, 8]. The NF- $\mathrm{kB}$ transcription factor is a dimmer in its active form, consisting of proteins from the REL family [9]. $\mathrm{NF}-\kappa \mathrm{B}$ is involved in the regulation of a wide array of distinct genes which are functionally involved in immune function, inflammation, differentiation, cell survival, growth, stress response and apoptosis [9]. Free radicals have also been functionally involved in the regulation of a wide panel of phosphatases or kinases, as well as in the regulation of the TGF- $\beta$ and the $H$-Ras oncogene [2].

In relation to proteome homeostasis (or proteostasis), the network of the oxidative-stress responsive transcription factors activate (among others) the molecular chaperones which represent key effectors of the heat shock and proteotoxic insult response. Molecular chaperones catalyze correct folding of nascent polypeptides, prevent protein misfolding and aggregation of nonenzymatically modified proteins (e.g. by advanced glycation end-products or oxidation), refold misfolded proteins, and target damaged proteins for degradation in the proteasome or lysosome [6]. The ubiquitin-proteasome and autophagylysosome degradation systems represent sites of removing both short-lived normal or damaged dysfunctioning proteins, thus exerting a tight control in the levels of regulatory proteins and in the maintenance of a functional proteome $[10,11]$.

\section{Oxidative Stress Implications in Ageing and Pathophysiology}

Organismal ageing affects most, if not all, tissues and organs of the body. It refers to a multifactorial process modulated by the interplay among environmental and genetic factors [12]. At the tissue and cellular level, ageing-related gradual accumulation of damage affects all types of biomolecules in postmitotic and mitotic cells, as well as at the extracellular matrix. According to the 'free radical theory of ageing', free radical accumulation during one's lifetime would lead to potentially deleterious by-products which unavoidably damage biomolecules and are thus responsible for degenerative diseases and 
ageing [1]. The direct implication of free radicals to longevity has been experimentally demonstrated by mutants of specific genes in invertebrates such as Caenorhabditis elegans and Drosophila melanogaster, which have been linked to increased lifespan due to reduced damage by oxidants [13]. Similarly, in mammals a mutant of the p $66^{\mathrm{SHC}}$ gene increased resistance to oxidative stress and prolonged lifespan [14], while in in vitro studies mammalian senescing cells are marked by higher levels of ROS and significant proteotoxic stress [15].

Beyond its effect in the processes of ageing, oxidative stress also plays a central role in various pathological conditions such as neurodegeneration, vascular and metabolic syndromes, as well as tumor formation [15-17]. Specifically, brain ischemia and stroke have been associated with free radical-mediated reactions [18], and there is a consensus that oxidative stress is an early event in Alzheimer's disease [19] and contributes to dopaminergic cell degeneration in Parkinson's disease [20]. Moreover, high endogenous levels of oxidative stress have been found is several tumors, including colorectal carcinoma, leukemia, and breast, ovarian and stomach cancer [17].

Sustained high levels of oxidants are tumorigenic as they can cause several DNA modifications, single- and double-strand DNA breaks, and oxidatively generated non-double-strand clustered DNA lesions. The two most common DNA base modifications are 8-oxodG (a marker of oxidative stress-related DNA damage) and 2,6-diamino-4-hydroxy-5-formamidopyrimidine [17]. Interestingly, there may be sites in the genome that are more susceptible to oxidative stress-related protumorigenic effects as a number of tumor suppressor genes have been identified as being silenced by oxidative-induced aberrant $\mathrm{CpG}$ island promoter methylation [21]. Oxidants are also responsible for the production of lipid aldehydes, such as 4-oxo-trans-2,3-nonenal (4-ONE) and 4-hydroxy-trans-2,3-nonenal (4-HNE) [22], which can disrupt membrane structure and can also react with various amino acids enabling protein carbonylation [23]. Heavily carbonylated proteins tend to form aggregates that are resistant to degradation and accumulate as unfolded or damaged proteins [23]. Such aggregates cause global proteome instability as they (among others) disrupt the functionality of the proteolytic pathways [24]. In conclusion, sustained high levels of oxidative stress will severely disrupt cellular homeostasis and particularly proteostasis by causing significant protein damage and proteotoxic stress. Thus, the role of the intra- and extracellular molecular chaperone network in safeguarding the proteome is critical.

\section{Apolipoprotein J/Clusterin (CLU)}

\section{CLU Gene Locus, Gene Regulation and Protein Isoforms}

The conventional translation product of the CLU gene in humans is encoded by an mRNA transcribed from a single copy gene, located at chromosome 8 and organized into nine exons [25]. The CLU gene exhibits an almost ubiquitous tissue expression pattern during both development and in adults, and encodes for a glycosylated secretory heterodimeric protein of approximately $75 \mathrm{kDa}$ (sCLU; hereafter CLU), which is secreted either constitutively or through regulated routes (fig. 1). It has been reported that apoptotic signals in human and rodent cells can induce the production of various CLU protein isoforms [16], most of which remain uncharacterized. Of these nonconventional isoforms, the most well-described variant refers to a stress-inducible nonglycosylated nucleocytosolic protein isoform of approximately $49 \mathrm{kDa}$ (nCLU) that is encoded by an alternatively spliced mRNA of the CLU gene locus and reportedly exerts a cytostatic and proapoptotic function [26].

Given the fact that the induction of the conventional CLU mRNA and protein (sCLU) induction are widespread phenomena in developmental and pathophysiological states (see below), it is anticipated that the CLU gene expression levels will be tightly regulated. Indeed, the CLU gene promoter is highly conserved and contains several regulatory elements that may regulate the complex tissue-specific control of the gene. These elements include SP1 and NF1 sites, as well as sites for AP-1 and a conserved 14-bp 'CLU-specific element', which is recognized by HSF1 and can mediate stress-induced transcription [27]. Moreover, CLU gene expression was found to be regulated by NF- $\kappa \mathrm{B}$; numerous growth factors and cytokines; oncogenes, and several stress- or apoptosis-inducing agents including heat shock, UVA, UVB, proteotoxic stress, heavy metals, oxidants, hyperoxia, ionizing radiation and chemotherapeutic drugs [16, 28]. Therefore, it seems that that by acting in synergy, the CLU-specific element and AP-1 elements make the CLU gene promoter particularly sensitive to even minute environmental or organismal changes.

Interestingly, the CLU gene is also regulated epigenetically as its promoter contains a CpG-rich methylation domain. More specifically, epigenetic factors downregulate CLU gene expression in retinal pigment epithelial cells [29], and hepatitis delta virus epigenetically enhances CLU expression via histone acetylation in human hepatocellular carcinoma cells [30]. Also, oncogenic H-Ras 
Fig. 1. Reported sites of conventional CLU (sCLU) localization and function (shown here in epithelial secretory cells). In the default pathway, sCLU mRNA is targeted to the ER for translation (1). The produced protein is processed to Golgi $(G)$ stacks and secreted in the apical (1') or basolateral (1") extracellular spaces. At the extracellular space, CLU may (among others) chaperone normal proteins (NP) or bind damaged dysfunctioning proteins (DP) and target them via receptor $(\mathrm{Re})$-mediated endocytosis (En) (2) to lysosome (L) for degradation. Reportedly, ER sCLU can target damaged proteins for ER-associated protein degradation (ERAD) via ER-attached proteasomes. Via a currently unknown mechanism, sCLU or its presecretory form (psCLU) may escape the secretory pathway $\left(3,3^{\prime}\right)$ and localize in the cytosol (Cyt). Considering that the protein has several localization motifs (including nuclear localization signals) on its sequence, it can then redistribute in the nucleus $(\mathrm{N})$ or to the mitochondria (M). Moreover, cytosolic sCLU can bind physiological normal protein complexes or target proteins for degradation to cytosolic proteasomes (P). Additional stress-induced CLU isoforms (not shown here) produced by alternative splicing can also localize in the nucleocytosolic compartments. SV = Secretory vesicle; $\mathrm{R}=$ ribosome.

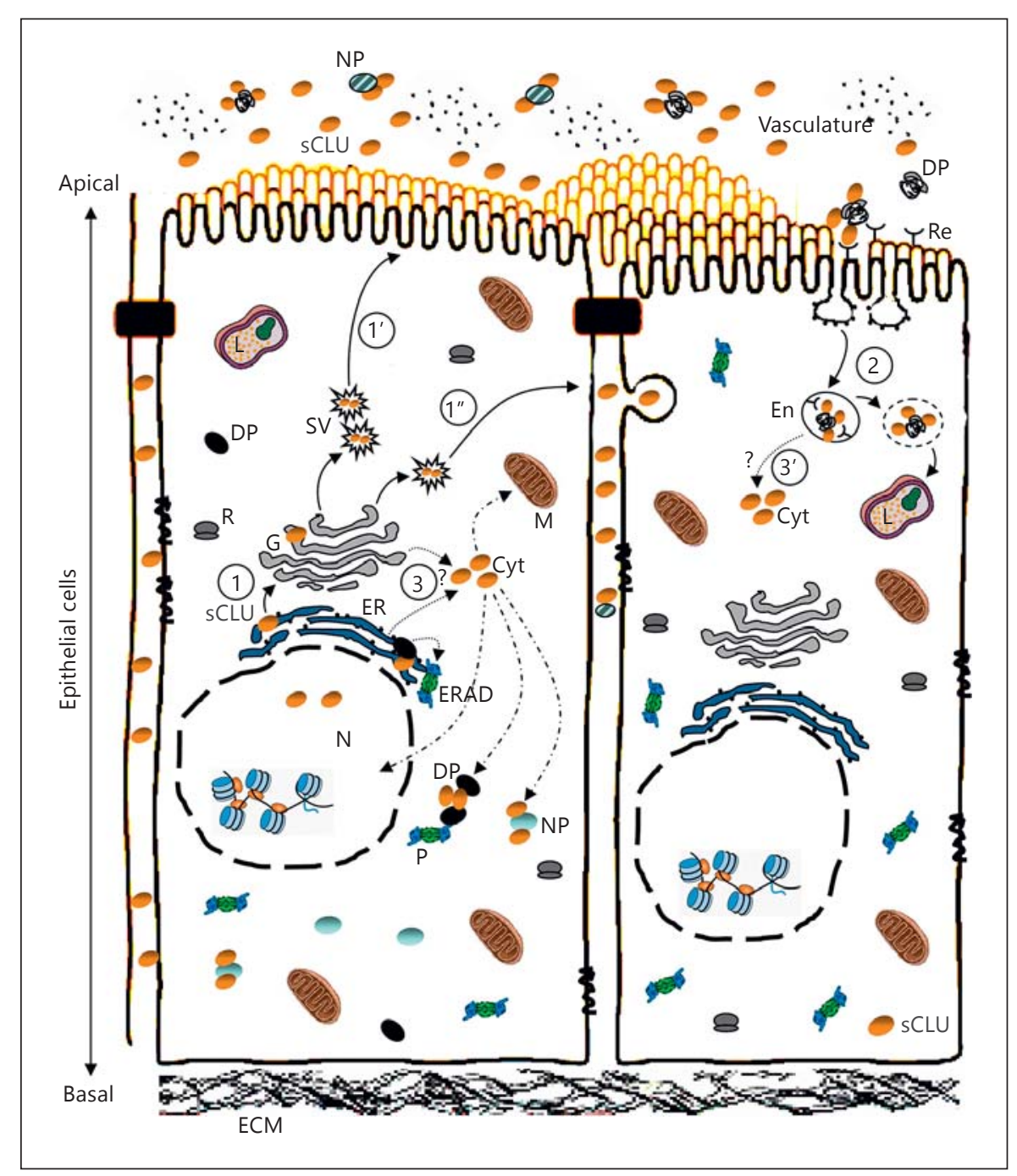

suppresses CLU expression in rat fibroblast cells in a promoter methylation-dependent manner [31], while the CLU gene promoter was found to be methylated in the TRAMP-C2 cell line and in the human prostate cancer cell line LNCaP [32]. Finally, the CLU gene was found to be silenced in tumor cells via promoter histone deacetylation [33] or by the polycomb protein histone methyltransferase EZH2 [34].

\section{CLU Extracellular/Intracellular Localization}

As mentioned earlier, the conventional (sCLU encoding) CLU mRNA is targeted to the endoplasmic reticulum (ER) for translation and from there it is constitutively secreted (fig. 1). In those cells featuring a regulated exocytotic pathway, CLU secretion may depend on the appropriate extracellular signal. On an opposite route, CLU and its binding cargo (e.g. damaged extracellular proteins) can be endocytosed and targeted to the lysosomes by mainly binding to the endocytic receptor gp330/megalin or to other unknown receptors (fig. 1; see also below).

Interestingly, it seems that in certain cell types, CLU can (via a currently unknown mechanism) escape the secretory pathway (fig. 1) and localize in additional intracellular compartments, namely the mitochondria, the nucleus and the cytosol [35-37]. In support, it was recently shown that GRP78 associates with CLU under ER-stress conditions to facilitate its retrotranslocation and redistribution to the mitochondria in prostate cancer [38]. It has also been proposed that in stressed cells the retrotranslocation of CLU from the ER to the cytosol is likely to occur through a mechanism similar to the ER-associated protein degradation (fig. 1) pathway [36]. A cyto- 
solic function of CLU also correlates with its reported implication in the regulation of the NF- $\kappa \mathrm{B}$ nuclear translocation and activity $[39,40]$, while among the recently identified intracellular CLU interacting proteins are the erythrocytic protein Band-3 [41] and the molecular chaperone HSP60 [42].

\section{Proposed Function(s) of CLU in the Maintenance of}

Extracellular/Intracellular Proteostasis

After several years of debate it has become a mainstream notion that because CLU represents an extracellular functional homologue of the small heat shock proteins due to its ability to bind to partially unfolded proteins and inhibit their aggregation and precipitation via an ATP-independent mechanism [43]. According to sequence analysis predictions, CLU contains three long regions of natively disordered or molten globule-like structures containing putative amphipathic $\alpha$-helices which give CLU the ability to bind to a variety of molecules. Interestingly, as the protein seems to also localize at intracellular compartments (see above), CLU may represent the only known chaperone with both extra- and intracellular activity.

Considering this functional property of CLU, its distinct sites of localization and the wide variety of unrelated binding partners, it is not surprising that CLU has been functionally implicated in numerous processes including sperm maturation, cell differentiation, complement regulation, lipid transportation, cellular debris clearance, development, phagocyte recruitment, cell aggregation, adhesion and cell-cell interactions, maintenance of the Alzheimer $\mathrm{A} \beta$ peptides solubility, DNA repair, NF- $\kappa \mathrm{B}$ signaling, cell cycle regulation, and cell death execution $[16,28]$.

It has been also found that in the extracellular milieu, CLU (along with other extracellular chaperones) mediates the recognition and disposal of damaged proteins via receptor-mediated endocytosis and lysosomal degradation [44] (fig. 1). Moreover, the A $\beta(1-40)$ peptide-formed small oligomers interact with CLU to form long-lived, stable complexes; therefore, CLU can influence both the aggregation and disaggregation of $A \beta(1-40)$ by sequestration of the $A \beta$ oligomers [45].

Finally, immunoaffinity chromatography analyses indicated that although a variety of proteins copurified with CLU from both stressed and control plasma, several proteins were uniquely present or were much more abundant when plasma was stressed by physiologically relevant stress (e.g. shear stress; approx. $36 \mathrm{dyn} / \mathrm{cm}^{2}$ at $37^{\circ} \mathrm{C}$ ) [46]. In line with this finding, CLU was found to bind oxidized proteins and be functionally involved in the clearance and removal of this oxidized/defected material in senescing human erythrocytes through vesiculation [41].

\section{Functional Implication of CLU in Ageing and Age-Related Diseases}

Considering the increase in oxidative and proteotoxic stress during ageing, it is not surprising that CLU expression levels have been found to increase in both cellular senescence and normal ageing of various tissues [16]. Newer findings adding to these observations have indicated a functional implication of CLU in human erythrocytes in the aged organism, as well as during erythrocyte senescence [47]. Similarly, CLU has been functionally involved in most age-related diseases including cardiovascular and metabolic syndromes, degenerative diseases, inflammation, and cancer $[16,28,48]$.

Specifically, CLU levels are increased during the early phase of new-onset myocardial infarction and vascular damage [49], while, in support of its cytoprotective role, intravenous CLU administration reduces myocardial infarct size in rats [50]. Also, it was found that TLR3 activation induced expression of cytoprotective and anti-inflammatory CLU by vascular smooth muscle cells to potentially counteract atherosclerotic pathology [51]. Regarding the CLU implication in metabolic syndromes, glucose was found to exert a positive regulation on CLU expression in hepatocytes [52], and CLU gene polymorphisms have been associated with type 2 diabetes mellitus [53].

Given the chaperone function of CLU, its role during neurodegeneration should be mainly cytoprotective. Indeed, CLU has been identified as a new significant genetic determinant in Alzheimer's disease [54], where it is thought that the association between amyloidogenic peptides and CLU contributes to limit $A \beta$ species misfolding and facilitates their clearance from the extracellular space [55]. Moreover, CLU content in the plasma is associated with atrophy of the entorhinal cortex, baseline disease severity and rapid clinical progression in Alzheimer's disease [56], as well as with longitudinal brain atrophy in mild cognitive impairment [57].

Reportedly, the average secretory CLU concentration in human plasma is $100 \pm 50 \mu \mathrm{g} / \mathrm{ml}$, while it is about 10 times higher in human seminal plasma $[58,59]$. CLU concentration increases in diabetes type 2 and during development of coronary heart disease or at myocardial infarction [60]. Similarly, in other more recent studies, plas- 
ma CLU level has been associated with the occurrence of coronary artery lesions in Kawasaki disease [61], and serum CLU levels $(52.8 \pm 0.8$ and $49.3 \pm 0.5 \mu \mathrm{g} / \mathrm{ml}$ in healthy Japanese men and women, respectively) have been found to be positively related to blood glucose content and also to increase in type 2 diabetic patients (men: $83.1 \pm$ $3.4 \mu \mathrm{g} / \mathrm{ml}$; women: $64.0 \pm 2.3$ ) [62]. Serum CLU concentration was also found to be increased in prostate cancer patients, where it was significantly associated with major prognostic factors other than biopsy Gleason score [63]; in patients with poorly differentiated hepatocellular carcinoma and lymph node infiltration [64], and in patients with bladder cancer [65]; in these latter patients, CLU concentration was also found to increase in urine [65].

Chronic inflammation induces (among others) increased oxidative stress affecting many different cell types, including stem cells [66], and thus represents an appropriate model to test CLU responsiveness to oxidant injury. It has been found that CLU expression levels correlated positively with most inflammatory diseases and virus infections [16]. More recently, CLU gene expression was found to be induced during progression of primary osteoarthritis [67]. Interestingly, CLU seems to also directly intervene in NF- $\kappa B$ regulation, although its role remains debated; depending on the experimental model, CLU had either a negative [39] or a positive effect [40] on NF- $\kappa \mathrm{B}$ activity.

CLU has been also functionally implicated in cancer, and its role in that disease has been extensively reviewed $[16,28,48]$. Specifically, CLU has been involved in all aspects of tumorigenesis including tumor formation and progression, metastasis and chemoresistance acquisition. It was recently reported that genetic inactivation of CLU in mice promoted transformation of the prostate epithelium in the majority of CLU knockout mice via the induction of NF-kB signaling [68]. In addition, c-Myc activation of miRNAs attenuates the TGF- $\beta$ signaling pathway to shut down CLU expression, thereby stimulating angiogenesis and tumor cell growth [69]. Although these studies added to previous findings suggesting a tumor-suppressing effect of CLU, CLU expression levels were positively correlated with disease progression in the majority of reported studies [16]. More recently, it was found that AKT exerted its cytoprotective role in prostate tumor cells via the induction of CLU [70], as well as that low-dose ionizing radiation-induced IGF-1 mediates CLU expression that interferes with TGF $\beta 1$ signaling to confer a prosurvival bystander effect [71]. Moreover, in prostate cancer cells, CLU mediated TGF- $\beta$-induced epithelial-mesenchymal transition and metastasis via Twist1 [72], while transcriptome profiling in a TGF$\beta$-induced epithelial-mesenchymal transition model revealed extracellular CLU as a target for therapeutic antibodies [73]. Finally, tumor-derived secretory CLU induced epithelial-mesenchymal transition and facilitated hepatocellular carcinoma metastasis [74], while the loss of expression of the tumor suppressor gene NKX3.1 in the early phases of prostate tumorigenesis correlated with CLU induction [75]. In this latter study, the authors also showed that in human prostate tissue samples, loss of NKX3.1 expression and corresponding CLU overexpression are colocalized at sites of prostatic inflammatory atrophy, a possible very early stage of human prostate tumorigenesis [75].

\section{CLU Regulation by Oxidative Stress}

The only common characteristic shared by normal ageing and most (if not all) of the aforementioned pathological conditions where CLU has been implicated is that they all represent states of increased oxidative injury. It was thus proposed recently that CLU is a sensitive cellular biosensor of oxidative stress that due to its chaperone activity functions to protect cells from the deleterious effects of oxidative and proteotoxic stress [16]. Specifically, it is anticipated that the presence of both the AP-1 and CLU-specific element regulatory elements in the CLU gene promoter [27] make the CLU gene an extremely sensitive biosensor to exogenous or endogenous stress and particularly to free radicals and their derivatives. Indeed, CLU was induced in human cells by ionizing radiation, proteotoxic stress, various oxidants, UVB and heavy metals [16].

Several studies have demonstrated that CLU has a cytoprotective role against the deleterious effects of oxidants. Specifically, CLU protected normal human fibroblasts from cytotoxicity induced by oxidants or UVB, human prostate cancer cells from oxidative stress-induced DNA damage, lung fibroblasts from cigarette smoke oxidants, and human retinal cells from free radical damage or in vitro ischemia; additionally, CLU protected human epidermoid cancer cells from $\mathrm{H}_{2} \mathrm{O}_{2}$, superoxide anion, hyperoxia and UVA [16]. In line with these findings, it was recently shown that CLU protected cardiomyocytes from oxidative stress-induced apoptosis via the Akt/ GSK-3 $\beta$ signaling pathway [76] and human corneal endothelial cells from oxidants [77]. The direct implication of CLU in the protection against oxidants was demonstrated in a very interesting in vivo study, where it was 


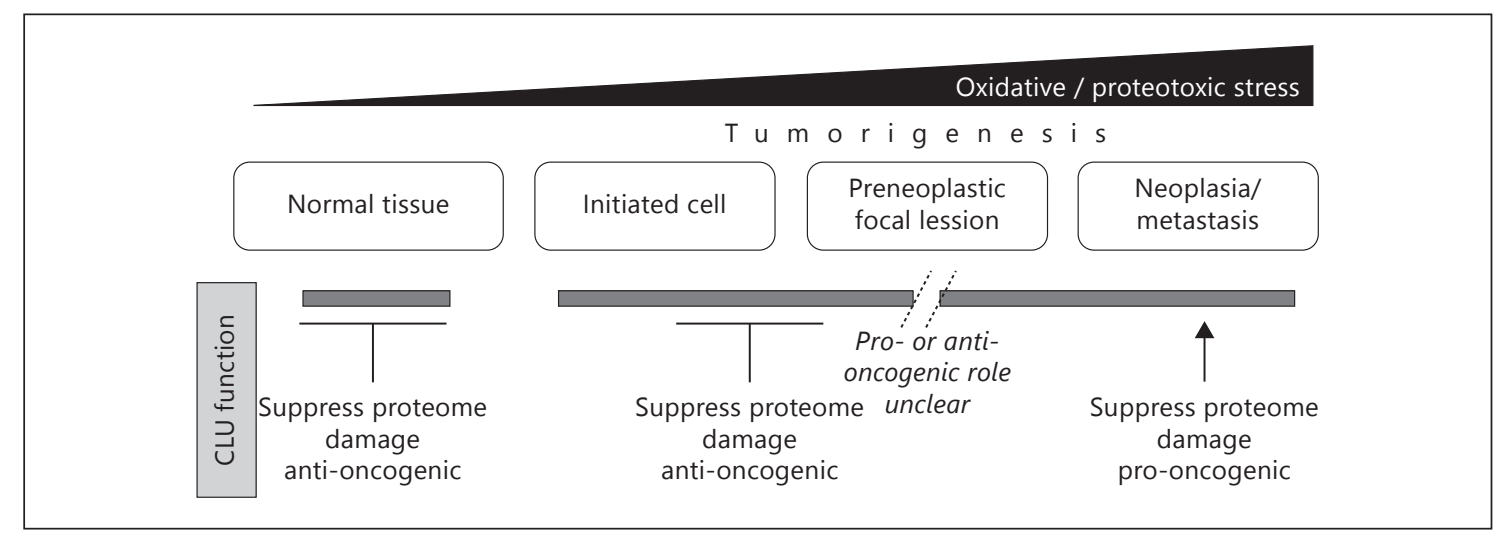

Fig. 2. Proposed implication of conventional CLU (sCLU) in carcinogenesis, a diseased cellular state of increased oxidative and proteotoxic stress. As the main function of sCLU relates to its potent chaperone activity, it is anticipated that sCLU will exert a cytoprotective function by contributing to proteostasis maintenance at both the extra- and, most likely, intracellular compartments. This property should be obviously antioncogenic as it will provide a barrier to oncogenic hits in both normal cells and (most likely) in early phases of cancer. In neoplasia and metastasis, how-

shown that overexpression of the conventional secretory human CLU (but not a truncated intracellular form) in Drosophila melanogaster increased resistance to heat shock, wet starvation and oxidative stress, and extended their lifespan [78]. The disulfide linkages of human CLU expressed in Drosophila might be involved in the reduction of total ROS levels and in increased stress tolerance since the antioxidant activity of CLU was abolished when the sulfhydryl groups of CLU cysteines were blocked with $\mathrm{N}$-ethylmaleimide. It was thus proposed by the authors that CLU functions as an antioxidant protein via its cysteine sulfhydryl groups to reduce ROS levels and delay organismal ageing in fruit flies [78]. This novel functional property of CLU indicates that it may also represent a unique module of both the proteostasis and antioxidant response networks.

\section{Concluding Remarks: Future Perspectives}

For quite some time the functional implication of CLU in critical cellular processes (e.g. cellular death or carcinogenesis) has been debated mainly due to contradictory findings in an array of various cell types and tissues $[16,28,48]$. So, considering that CLU is a novel extra- and, most likely, intracellular chaperone, can the paradox be resolved? ever, the chaperone activity of sCLU will confer significant survival advantage to tumor cells. This latter assumption also explains the selective pressure for higher expression levels or de novo synthesis of sCLU in advanced cancer (our unpubl. findings). Given the complex regulation of the gene and its responsiveness to most stressors (see text), a number of additional parameters, such as the type of tissue, the local microenvironment and the oncogenic pathways activated, may define the role of sCLU in tumor pathophysiology.

First of all, as described above, it is reasonable to argue that the CLU gene is a sensitive biosensor of environmental insults and, more specifically, oxidative stress that is the driving force of most, if not all, age-related diseases where CLU has been functionally implicated. As the main role of CLU in these pathological conditions would be to cease the deleterious effects of oxidative (demonstrated also by the studies in transgenic flies overexpressing CLU [78]) and proteotoxic stress, it is anticipated that the main effect(s) of CLU during the progression of a disease would initially be benign. For instance, if we consider cancer, at the initial stages of the disease the function of CLU would be tumor suppressive as it will decrease proteome damage and thus, indirectly, suppress tumor progression. Age- or lifestyle-related proteome damage (e.g. protein oxidation) alters protein function and may promote tumorigenesis by resulting in dysfunctioning protein machines that gradually become ineffective in removing stressors and also in performing a number of cellular functions that are critical for genomic stability, such as DNA replication and/or repair. Nevertheless, at advanced stages of neoplasia and/or metastasis, CLU may become a potent oncogene because it will offer a significant survival advantage in tumor cells (fig. 2). This biphasic function was particularly demonstrated during skin carcinogenesis were CLU was a tumor attenuator in early carcinogenesis and an enhancer 
of the malignant phenotype in late carcinogenesis [79]. Additionally, it is anticipated that even in those cases where normal or tumor-initiated cells do not express CLU, there would be a selective (Darwinian) pressure for higher CLU expression levels as cancer progress. Indeed, it seems that in some tumors the suppressing mechanisms can be bypassed allowing cells to progress to de novo CLU gene expression in the established tumor [80; our unpubl. observations].

On the basis of this view, high expression levels of CLU in advanced cancer should offer a significant therapeutic window. This possibility is currently being exploited in clinical trials [81] by the successful paradigm of OGX-011, a 2'-methoxyethyl-modified phosphorothioate antisense oligonucleotide that is complementary to CLU mRNA; work in progress aims to finalize plans for two phase III clinical studies. Additional translational applications in relation to CLU biology may include peptides that bind and inhibit CLU in vivo [82] or molecular imaging of tumors by using CLU-binding fluorescent peptides [83].

Given the significant biomedical interest for the CLU biological function(s), a number of topics in the CLU research field need prompt investigation. These include (1) the understanding of the CLU functional role in mitochondria or in other organelles; (2) its functional im- plication in cell signaling pathways, including the possible gene regulation by microRNAs; (3) the understanding of the regulation and localization of the various protein isoforms, and (4) the identification of novel binding partners under basal conditions or in stress and diseases. Moreover, it would be essential to develop mammalian transgenic CLU models and to elucidate the CLU protein crystal structure. Clear progress in these issues will add to the existing knowledge and to our efforts to understand the function of this most fascinating protein.

\section{Acknowledgements}

I apologize to those investigators whose work, due to space constrains, was not cited. This review and the research studies by the group of the author are supported by the following institutions and grants: the University of Athens SARG, the Bodosakis Foundation, INsPiRE (EU, REGPOT-CT-2011-284460), Greece-France R\&T Cooperation Grant (Hellenic GSRT, 09FR-56), and the EU COST actions CM1001 and CM1004.

\section{Disclosure Statement}

The author declares that no competing financial interests exist.

\section{References}

1 Beckman KB, Ames BN: The free radical theory of aging matures. Physiol Rev 1998;78: 547-581.

2 Martindale JL, Holbrook NJ: Cellular response to oxidative stress: signaling for suicide and survival. J Cell Physiol 2002;192:1-15.

$>3$ Klaunig JE, Kamendulis LM: The role of oxidative stress in carcinogenesis. Annu Rev Pharmacol Toxicol 2004;44:239-267.

4 Valko M, Rhodes CJ, Moncol J, Izakovic M, Mazur M: Free radicals, metals and antioxidants in oxidative stress-induced cancer. Chem Biol Interact 2006;160:1-40.

$>5$ Sykiotis GP, Bohmann D: Stress-activated cap'n'collar transcription factors in aging and human disease. Sci Signal 2010;3:re3.

$\checkmark 6$ Soti C, Pal C, Papp B, Csermely P: Molecular chaperones as regulatory elements of cellular networks. Curr Opin Cell Biol 2005;17: 210-215.

7 Shaulian E, Karin M: AP-1 in cell proliferation and survival. Oncogene 2001;20:23902400.

$>8$ Vousden KH, Lane DP: p53 in health and disease. Nat Rev Mol Cell Biol 2007;8:275283.
>9 Karin M, Greten FR: NF- $\mathrm{BB}$ : linking inflammation and immunity to cancer development and progression. Nat Rev Immunol 2005;5: 749-759.

10 Poppek D, Grune T: Proteasomal defense of oxidative protein modifications. Antioxid Redox Signal 2006;8:173-184.

11 Kaushik S, Cuervo AM: Chaperones in autophagy. Pharmacol Res 2012;66:484-493.

12 Kirkwood TB: Evolution of ageing. Mech Ageing Dev 2002;123:737-745.

13 Hekimi S, Guarente L: Genetics and the specificity of the aging process. Science 2003;299: 1351-1354.

14 Migliaccio E, Giorgio M, Mele S, Pelicci G, Reboldi P, Pandolfi PP, Lanfrancone L, Pelicci PG: The p66shc adaptor protein controls oxidative stress response and life span in mammals. Nature 1999;402:309-313.

15 Grune T, Jung T, Merker K, Davies KJ: Decreased proteolysis caused by protein aggregates, inclusion bodies, plaques, lipofuscin, ceroid, and 'aggresomes' during oxidative stress, aging, and disease. Int J Biochem Cell Biol 2004;36:2519-2530.
16 Trougakos IP, Gonos ES: Chapter 9: oxidative stress in malignant progression: the role of Clusterin, a sensitive cellular biosensor of free radicals. Adv Cancer Res 2009;104:171-210.

17 Sesti F, Tsitsilonis OE, Kotsinas A, Trougakos IP: Oxidative stress-mediated biomolecular damage and inflammation in tumorigenesis. In Vivo 2012;26:395-402.

18 Alexandrova M, Bochev P, Markova V, Bechev B, Popova M, Danovska M, Simeonova V: Dynamics of free radical processes in acute ischemic stroke: influence on neurological status and outcome. J Clin Neurosci 2004;11: 501-506.

19 Zhu X, Raina AK, Perry G, Smith MA: Alzheimer's disease: the two-hit hypothesis. Lancet Neurol 2004;3:219-226.

20 Jenner P: Oxidative stress in Parkinson's disease. Ann Neurol 2003;53:S26-S36.

21 Ushijima T: Detection and interpretation of altered methylation patterns in cancer cells. Nat Rev Cancer 2005;5:223-231.

22 Esterbauer H, Schaur RJ, Zollner H: Chemistry and biochemistry of 4-hydroxynonenal, malonaldehyde and related aldehydes. Free Radic Biol Med 1991;11:81-128. 
-23 Dalle-Donne I, Aldini G, Carini M, Colombo R, Rossi R, Milzani A: Protein carbonylation, cellular dysfunction, and disease progression. J Cell Mol Med 2006;10:389-406.

24 Grune T, Davies KJ: The proteasomal system and HNE-modified proteins. Mol Aspects Med 2003;24:195-204.

25 Wong P, Taillefer D, Lakins J, Pineault J, Chader G, Tenniswood M: Molecular characterization of human TRPM-2/clusterin, a gene associated with sperm maturation, apoptosis and neurodegeneration. Eur J Biochem 1994; 221:917-925.

-26 Leskov KS, Araki S, Lavik JP, Gomez JA, Gama V, Gonos ES, Trougakos IP, Matsuyama S, Boothman DA: CRM1 protein-mediated regulation of nuclear clusterin (nCLU), an ionizing radiation-stimulated, Bax-dependent pro-death factor. J Biol Chem 2011;286:40083-40090.

27 Michel D, Chatelain G, Herault Y, Brun G: The expression of the avian clusterin gene can be driven by two alternative promoters with distinct regulatory elements. Eur J Biochem 1995;229:215-223.

-28 Zoubeidi A, Gleave M: Small heat shock proteins in cancer therapy and prognosis. Int $\mathrm{J}$ Biochem Cell Biol 2012;44:1646-1656.

-29 Suuronen T, Nuutinen T, Ryhänen T, Kaarniranta K, Salminen A: Epigenetic regulation of clusterin/apolipoprotein J expression in retinal pigment epithelial cells. Biochem Biophys Res Commun 2007;357:397-401.

30 Liao FT, Lee YJ, Ko JL, Tsai CC, Tseng CJ, Sheu GT: Hepatitis delta virus epigenetically enhances clusterin expression via histone acetylation in human hepatocellular carcinoma cells. J Gen Virol 2009;90:1124-1134.

- 31 Lund P, Weisshaupt K, Mikeska T, Jammas D, Chen X, Kuban RJ, Ungethüm U, Krapfenbauer U, Herzel HP, Schäfer R, Walter J, Sers C: Oncogenic HRAS suppresses clusterin expression through promoter hypermethylation. Oncogene 2006;25:4890-4903.

- 32 Rauhala HE, Porkka KP, Saramäki OR, Tammela TL, Visakorpi T: Clusterin is epigenetically regulated in prostate cancer. Int J Cancer 2008; 123:1601-1609.

-33 Hellebrekers DM, Melotte V, Viré E, Langenkamp E, Molema G, Fuks F, Herman JG, Van Criekinge W, Griffioen AW, van Engeland M: Identification of epigenetically silenced genes in tumor endothelial cells. Cancer Res 2007; 67:4138-4148.

-34 Wang C, Liu Z, Woo CW, Li Z, Wang L, Wei JS, Marquez VE, Bates SE, Jin Q, Khan J, Ge $\mathrm{K}$, Thiele CJ: EZH2 Mediates epigenetic silencing of neuroblastoma suppressor genes CASZ1, CLU, RUNX3, and NGFR. Cancer Res 2012;72:315-324.

- 35 Zhang H, Kim JK, Edwards CA, Xu Z, Taichman R, Wang CY: Clusterin inhibits apoptosis by interacting with activated Bax. Nat Cell Biol 2005;7:909-915.

- 36 Nizard P, Tetley S, Le Dréan Y, Watrin T, Le Goff P, Wilson MR, Michel D: Stress-induced retrotranslocation of clusterin/ApoJ into the cytosol. Traffic 2007;8:554-565.
37 Trougakos IP, Lourda M, Antonelou MH, Kletsas D, Gorgoulis VG, Papassideri IS, Zou Y, Margaritis LH, Boothman DA, Gonos ES: Intracellular clusterin inhibits mitochondrial apoptosis by suppressing $\mathrm{p} 53$-activating stress signals and stabilizing the cytosolic Ku70-Bax protein complex. Clin Cancer Res 2009;15: 48-59.

38 Li N, Zoubeidi A, Beraldi E, Gleave ME: GRP78 regulates clusterin stability, retrotranslocation and mitochondrial localization under ER stress in prostate cancer. Oncogene 2013;32:1933-1942.

39 Essabbani A, Margottin-Goguet F, Chiocchia G: Identification of clusterin domain involved in NF-kappaB pathway regulation. J Biol Chem 2010;285:4273-4277.

40 Zoubeidi A, Ettinger S, Beraldi E, Hadaschik B, Zardan A, Klomp LW, Nelson CC, Rennie PS, Gleave ME: Clusterin facilitates COMMD1 and I-kappaB degradation to enhance NFkappaB activity in prostate cancer cells. Mol Cancer Res 2010;8:119-130.

-41 Antonelou MH, Kriebardis AG, Stamoulis KE, Trougakos IP, Papassideri IS: Apolipoprotein J/clusterin in human erythrocytes is involved in the molecular process of defected material disposal during vesiculation. PLoS One 2011;6:e26033.

42 Chaiwatanasirikul KA, Sala A: The tumoursuppressive function of CLU is explained by its localisation and interaction with HSP60. Cell Death Dis 2011;2:e219.

43 Wyatt A, Yerbury J, Poon S, Dabbs R, Wilson M: Chapter 6: the chaperone action of clusterin and its putative role in quality control of extracellular protein folding. Adv Cancer Res 2009;104:89-114.

44 Wyatt AR, Yerbury JJ, Berghoffer P, Greguric I, Katsifis A, Dobson CM, Wilson MR: Clusterin facilitates in vivo clearance of extracellular misfolded proteins. Cell Mol Life Sci 2011; 68:3919-3931.

45 Narayan P, Orte A, Clarke RW, Bolognesi B, Hook S, Ganzinger K, Meehan S, Wilson MR, Dobson C, Klenerman D: The extracellular chaperone clusterin sequesters oligomeric forms of the Alzheimer's A $\beta$ peptide. Nat Struct Mol Biol 2011;19:79-83.

46 Wyatt AR, Wilson MR: Identification of human plasma proteins as major clients for the extracellular chaperone clusterin. J Biol Chem 2010;285:3532-3539.

47 Antonelou MH, Kriebardis AG, Stamoulis KE, Trougakos IP, Papassideri IS: Apolipoprotein J/clusterin is a novel structural component of human erythrocytes and a biomarker of cellular stress and senescence. PLOS One 2011;6:e26032.

48 Sala A, Bettuzzi S, Pucci S, Chayka O, Dews M, Thomas-Tikhonenko A: Regulation of CLU gene expression by oncogenes and epigenetic factors implications for tumorigenesis. Adv Cancer Res 2009;105:115-132.
49 Cubedo J, Padró T, García-Moll X, Pintó X, Cinca J, Badimon L: Proteomic signature of apolipoprotein $\mathrm{J}$ in the early phase of new-onset myocardial infarction. J Proteome Res 2011;10:211-220.

50 Van Dijk A, Vermond RA, Krijnen PA, Juffermans LJ, Hahn NE, Makker SP, Aarden LA, Hack E, Spreeuwenberg M, van Rossum BC, Meischl C, Paulus WJ, Van Milligen FJ, Niessen HW: Intravenous clusterin administration reduces myocardial infarct size in rats. Eur J Clin Invest 2010;40:893-902.

51 Baiersdörfer M, Schwarz M, Seehafer K, Lehmann C, Heit A, Wagner H, Kirschning CJ, Koch-Brandt C: Toll-like receptor 3 mediates expression of clusterin/apolipoprotein $\mathrm{J}$ in vascular smooth muscle cells stimulated with RNA released from necrotic cells. Exp Cell Res 2010;316:3489-3500.

52 Kim G, Kim GH, Oh GS, Yoon J, Kim HW, Kim MS, Kim SW: SREBP-1c regulates glucose-stimulated hepatic clusterin expression. Biochem Biophys Res Commun 2011;408: 720-725.

53 Daimon M, Oizumi T, Karasawa S, Kaino W, Takase K, Tada K, Jimbu Y, Wada K, Kameda W, Susa S, Muramatsu M, Kubota I, Kawata $\mathrm{S}$, Kato T: Association of the clusterin gene polymorphisms with type 2 diabetes mellitus. Metabolism 2011;60:815-822.

54 Lambert JC, Amouyel P: Genetics of Alzheimer's disease: new evidences for an old hypothesis? Curr Opin Genet Dev 2011;21: 295-301.

55 Charnay Y, Imhof A, Vallet PG, Kovari E, Bouras C, Giannakopoulos P: Clusterin in neurological disorders: molecular perspectives and clinical relevance. Brain Res Bull 2012;88:434-443.

56 Thambisetty M, Simmons A, Velayudhan L, Hye A, Campbell J, Zhang Y, Wahlund LO, Westman E, Kinsey A, Güntert A, Proitsi P, Powell J, Causevic M, Killick R, Lunnon K, Lynham S, Broadstock M, Choudhry F, Howlett DR, Williams RJ, Sharp SI, Mitchelmore C, Tunnard C, Leung R, Foy C, O'Brien D, Breen G, Furney SJ, Ward M, Kloszewska I, Mecocci P, Soininen H, Tsolaki M, Vellas B, Hodges A, Murphy DG, Parkins S, Richardson JC, Resnick SM, Ferrucci L, Wong DF, Zhou Y, Muehlboeck S, Evans A, Francis PT, Spenger C, Lovestone S: Association of plasma clusterin concentration with severity, pathology, and progression in Alzheimer disease. Arch Gen Psychiatry 2010;67:739-748.

57 Thambisetty M, An Y, Kinsey A, Koka D, Saleem M, Güntert A, Kraut M, Ferrucci L, Davatzikos C, Lovestone S, Resnick SM: Plasma clusterin concentration is associated with longitudinal brain atrophy in mild cognitive impairment. Neuroimage 2012;59:212-217.

58 Murphy BF, Kirszbaum L, Walker ID, d'Apice AJ: SP-40,40, a newly identified normal human serum protein found in the SC5b-9 complex of complement and in the immune deposits in glomerulonephritis. J Clin Invest 1988;81:1858-1864. 
59 Jenne DE, Lowin B, Peitsch MC, Böttcher A, Schmitz G, Tschopp J: Clusterin (complement lysis inhibitor) forms a high density lipoprotein complex with apolipoprotein A-I in human plasma. J Biol Chem 1991;266: 11030-11036.

-60 Trougakos IP, Poulakou M, Stathatos M, Chalikia A, Melidonis A, Gonos ES: Serum levels of the senescence biomarker clusterin/ apolipoprotein $J$ increase significantly in diabetes type II and during development of coronary heart disease or at myocardial infarction. Exp Gerontol 2002;37:1175-1187.

-61 Yu HR, Kuo HC, Huang EY, Liang CD, Hwang KP, Lin IC, Sheen JM, Wang TJ, Wang CL, Yang KD: Plasma clusterin levels in predicting the occurrence of coronary artery lesions in patients with Kawasaki disease. Pediatr Cardiol 2010;31:1151-1156.

-62 Kujiraoka T, Hattori H, Miwa Y, Ishihara M, Ueno T, Ishii J, Tsuji M, Iwasaki T, Sasaguri Y, Fujioka T, Saito S, Tsushima M, Maruyama T, Miller IP, Miller NE, Egashira T: Serum apolipoprotein $\mathrm{J}$ in health, coronary heart disease and type 2 diabetes mellitus. J Atheroscler Thromb 2006;13:314-322.

63 Miyake H, Muramaki M, Furukawa J, Kurahashi T, Fujisawa M: Serum level of clusterin and its density in men with prostate cancer as novel biomarkers reflecting disease extension. Urology 2010;75:454-459.

64 Nafee AM, Pasha HF, Abd El Aal SM, Mostafa NA: Clinical significance of serum clusterin as a biomarker for evaluating diagnosis and metastasis potential of viral-related hepatocellular carcinoma. Clin Biochem 2012;45: 1070-1074.

-65 Stejskal D, Fiala RR: Evaluation of serum and urine clusterin as a potential tumor marker for urinary bladder cancer. Neoplasma 2006; 53:343-346.

66 Lepperdinger G: Inflammation and mesenchymal stem cell aging. Curr Opin Immunol 2011;23:518-524.

67 Fandridis E, Apergis G, Korres DS, Nikolopoulos K, Zoubos AB, Papassideri I, Trougakos IP: Increased expression levels of apolipoprotein $\mathrm{J} /$ clusterin during primary osteoarthritis. In Vivo 2011;25:745-749.
68 Bettuzzi S, Davalli P, Davoli S, Chayka O, Rizzi F, Belloni L, Pellacani D, Fregni G, Astancolle S, Fassan M, Corti A, Baffa R, Sala A: Genetic inactivation of ApoJ/clusterin: effects on prostate tumourigenesis and metastatic spread. Oncogene 2009;28:4344-4352.

69 Dews M, Fox JL, Hultine S, Sundaram P, Wang W, Liu YY, Furth E, Enders GH, ElDeiry W, Schelter JM, Cleary MA, ThomasTikhonenko A: The myc-miR-17 92 axis blunts TGF $\{$ beta $\}$ signaling and production of multiple TGF $\{$ beta $\}$-dependent antiangiogenic factors. Cancer Res 2010;70:8233-8246.

70 Zhong B, Sallman DA, Gilvary DL, Pernazza D, Sahakian E, Fritz D, Cheng JQ, Trougakos I, Wei S, Djeu JY: Induction of clusterin by AKT - role in cytoprotection against docetaxel in prostate tumor cells. Mol Cancer Ther 2010;9:1831-1841.

71 Klokov D, Leskov K, Araki S, Zou Y, Goetz EM, Luo X, Willson D, Boothman DA: Low dose IR-induced IGF-1-sCLU expression: a p53-repressed expression cascade that interferes with TGF $\beta 1$ signaling to confer a prosurvival bystander effect. Oncogene 2013;32: 479-490.

72 Shiota M, Zardan A, Takeuchi A, Kumano M, Beraldi E, Naito S, Zoubeidi A, Gleave ME: Clusterin mediates TGF- $\beta$-induced epithelial-mesenchymal transition and metastasis via Twist1 in prostate cancer cells. Cancer Res 2012;72:5261-5272.

73 Lenferink AE, Cantin C, Nantel A, Wang E, Durocher Y, Banville M, Paul-Roc B, Marcil A, Wilson MR, O'Connor-McCourt MD: Transcriptome profiling of a TGF-beta-induced epithelial-to-mesenchymal transition reveals extracellular clusterin as a target for therapeutic antibodies. Oncogene 2010;29:831-844.

74 Wang C, Jiang K, Kang X, Gao D, Sun C, Li Y, Sun L, Zhang S, Liu X, Wu W, Yang P, Guo K, Liu Y: Tumor-derived secretory clusterin induces epithelial-mesenchymal transition and facilitates hepatocellular carcinoma metastasis. Int J Biochem Cell Biol 2012;44:2308-2320.

75 Song $\mathrm{H}$, Zhang B, Watson MA, Humphrey PA, Lim H, Milbrandt J: Loss of Nkx3.1 leads to the activation of discrete downstream target genes during prostate tumorigenesis. Oncogene 2009;28:3307-3319.
6 Jun HO, Kim DH, Lee SW, Lee HS, Seo JH, Kim JH, Kim JH, Yu YS, Min BH, Kim KW: Clusterin protects $\mathrm{H} 9 \mathrm{c} 2$ cardiomyocytes from oxidative stress-induced apoptosis via Akt/ GSK-3 $\beta$ signaling pathway. Exp Mol Med 2011;43:53-61.

77 Shin YJ, Kim JH, Seo JM, Lee SM, Hyon JY, Yu YS, Wee WR: Protective effect of clusterin on oxidative stress-induced cell death of human corneal endothelial cells. Mol Vis 2009; 15:2789-2795.

78 Lee YN, Shim YJ, Kang BH, Park JJ, Min BH: Over-expression of human clusterin increases stress resistance and extends lifespan in Drosophila melanogaster. Biochem Biophys Res Commun 2012;420:851-856.

79 Thomas-Tikhonenko A, Viard-Leveugle I, Dews M, et al: Myc-transformed epithelial cells down-regulate clusterin, which inhibits their growth in vitro and carcinogenesis in vivo. Cancer Res 2004;64:3126-3136.

80 Andersen CL, Schepeler T, Thorsen K, Birkenkamp-Demtröder K, Mansilla F, Aaltonen LA, Laurberg S, Ørntoft TF: Clusterin expression in normal mucosa and colorectal cancer. Mol Cell Proteomics 2007;6:1039-1048.

81 Saad F, Hotte S, North S, Eigl B, Chi K, Czaykowski P, Wood L, Pollak M, Berry S, Lattouf JB, Mukherjee SD, Gleave M, Winquist E: Randomized phase II trial of Custirsen (OGX-011) in combination with docetaxel or mitoxantrone as second-line therapy in patients with metastatic castrate-resistant prostate cancer progressing after first-line docetaxel: CUOG trial P-06c. Clin Cancer Res 2011;17:5765-5773.

82 Kliger Y, Levy O, Oren A, Ashkenazy H, Tiran Z, Novik A, Rosenberg A, Amir A, Wool A, Toporik A, Schreiber E, Eshel D, Levine Z, Cohen Y, Nold-Petry C, Dinarello CA, Borukhov I: Peptides modulating conformational changes in secreted chaperones: from in silico design to preclinical proof of concept. Proc Natl Acad Sci USA 2009;106:13797-13801.

83 Filfil R, Paul-Roc B, Cantin C, Iqbal U, Tolkatchev D, Vinogradova A, Xu P, Ni F, O'Connor-McCourt MD, Lenferink AE: Molecular imaging of breast tumors using a near-infrared fluorescently labeled clusterin binding peptide. Int J Cancer 2012;131:E681-E692. 